\title{
Methods for Atomistic Simulations of Linear and Nonlinear Damping in Nanomechanical Resonators
}

\author{
Zahra Nourmohammadi, Sankha Mukherjee, Surabhi Joshi, \\ Jun Song, and Srikar Vengallatore \\ Journal of Microelectromechanical Systems, (Volume:24, Issue: 5) (2015) \\ doi: 10.1109/JMEMS.2015.2411747 \\ http://ieeexplore.ieee.org/xpl/articleDetails.jsp?arnumber $=7065227$ \\ Copyright 2015 IEEE. Personal use of this material is permitted. Permission \\ from IEEE must be obtained for all other users, including reprinting/ \\ republishing this material for advertising or promotional purposes, creating new \\ collective works for resale or redistribution to servers or lists, or reuse of any \\ copyrighted components of this work in other works
}




\title{
Methods for Atomistic Simulations of Linear and Nonlinear Damping in Nanomechanical Resonators
}

\author{
Zahra Nourmohammadi, Sankha Mukherjee, Surabhi Joshi, Jun Song, and Srikar Vengallatore
}

\begin{abstract}
Atomistic simulations can be used to compute damping from first principles and gain unprecedented insights into the mechanisms of dissipation. However, the technique is still in its infancy and many foundational aspects remain unexplored. As a step towards addressing these issues, we present here a comparative study of five different methods for estimating damping under isothermal conditions. Classical molecular dynamics was used to simulate the fundamental longitudinalmode oscillations of nanowires and nanofilms of silicon and nickel at room temperature $(300 \mathrm{~K})$ in the canonical ensemble using the Nosé-Hoover thermostat. In the sub-resonant regime, damping was quantified using the loss tangent and loss factor during steady-state harmonic vibration. The quality factor was obtained by analyzing the spectrum of thermomechanical noise and also from the Duffing-like nonlinearity in the frequency response under harmonic excitation. In addition, the nonlinear logarithmic decrement was obtained from the Hilbert transform of freely-decaying oscillations. We discuss the factors that must be considered while selecting simulation parameters; establish criteria for convergence and linearity; and highlight the relative merits and limitations of each method.
\end{abstract}

Index Terms-Damping, dissipation, molecular dynamics, MEMS, NEMS, quality factor.

\section{INTRODUCTION}

A LL vibrating structures dissipate energy by damping. The various sources of dissipation have been studied extensively using continuum thermomechanics and phenomenological models. Despite considerable effort, however, it has proved difficult to obtain a detailed atomic-

Manuscript received August 22, 2014; revised December 21, 2014; accepted March 6, 2015. This work was supported in part by the Natural Sciences and Engineering Research Council of Canada and in part by Compute Canada. Subject Editor A. Seshia.

Z. Nourmohammadi, S. Mukherjee, S. Joshi, and S. Vengallatore are with the Department of Mechanical Engineering, McGill University, Montreal, QC, H3A 0C3, Canada (email: zahra.nourmohammadi@mail.mcgill.ca; sankha.mukherjee@mail.mcgill.ca;surabhi.joshi@mail.mcgill.ca; srikar.vengallatore@mcgill.ca).

J. Song is with the Department of Mining and Materials Engineering, McGill University, Montreal, QC, H3A 0E8, Canada (email: jun.song2@mcgill.ca).

Z. Nourmohammadi and S. Mukherjee contributed equally to this work. Color versions of one or more of the figures in this paper are available online at http://ieeexplore.ieee.org. level understanding of the numerous mechanisms by which mechanical energy is converted into thermal energy (see, for example, [1]-[4]). In this context, a remarkable recent development is the emergence of a fundamentally new approach for studying material damping using large-scale atomistic simulations. Such simulations can provide unprecedented insights into the mechanisms of dissipation; predict the magnitude of damping from first-principles; and guide the design of resonant microelectromechanical systems (MEMS) and nanoelectromechanical systems (NEMS).

Classical molecular dynamics (MD) is a powerful and versatile technique for atomistic simulations in which atoms are modeled as point particles that evolve in time according to Newton's equations of motion. Material and structural properties are estimated by invoking ergodicity which states that time averages are equal to ensemble averages. Typically, the time evolution of a single ensemble is simulated to characterize the system [5]. Using this approach, classical MD has been used to explore dissipation in a small set of materials (including silicon [6], quartz [6], carbon nanotubes [7]-[11], graphene [12], [13], and silver [14]) using the microcanonical $N V E$ ensemble wherein the number of atoms $(N)$, volume $(V)$, and total energy $(E)$ are maintained constant. The microcanonical ensemble is well suited for simulating the exchange of energy between different mechanical modes (including, for example, energy conversion from a collective mode of mechanical vibrations to internal energy), but the average temperature of the structure increases steadily due to damping because the structure is isolated from its thermal environment [8].

In 2011, Kunal and Aluru [15] introduced an approach for MD simulations of damping under isothermal conditions by employing the canonical NVT ensemble to explore Akhiezer damping in nickel. The temperature $(T)$ was controlled by coupling the structure to the thermal reservoir using the NoséHoover thermostat [16], [17], and damping was quantified by computing the work done per cycle of oscillation [15]. Isothermal simulations are of great interest because they match the typical operating conditions of MEMS and NEMS, but the technique is still at an early stage of development.

As a step towards developing the technique, we present here a comparative study of five different methods for computing damping using the canonical ensemble. The various methods are as follows: (i) estimate dissipation using the loss factor, 
$\eta \equiv \Delta W / 2 \pi W$ (or, equivalently, the specific damping capacity, $\psi \equiv \Delta W / W=2 \pi \eta)$ where $\Delta W$ is the energy dissipated per cycle of vibration and $W$ is the maximum elastic energy during the vibration cycle; (ii) analyze steady-state time-harmonic oscillations to estimate damping in terms of the loss tangent, $\tan \phi$, where $\phi$ is the phase angle by which the force leads the displacement; (iii) estimate damping from thermomechanical noise by fitting the power spectral density (PSD) of displacement fluctuations to extract the quality factor (Q); (iv) analyze the frequency response to estimate the quality factor $(Q)$ at resonance; and (v) analyze the freely decaying oscillations of the structure to obtain the logarithmic decrement, $\delta$. The loss factor, specific damping capacity, and loss tangent are well suited for characterizing damping in the sub-resonant regime (that is, at frequencies less than the fundamental natural frequency, $\left.f_{0}\right)$. The other two measures $(Q$ and $\delta$ ) are used to quantify damping at the natural frequency.

The methods are demonstrated by using nickel and silicon as model materials. Damping is computed at room temperature (300 K) for the fundamental longitudinal-mode oscillations of nanofilms and nanowires of $\mathrm{Si}$ and $\mathrm{Ni}$. In all cases, the main goals are to establish robust protocols for selecting simulation parameters; establish criteria for convergence and linearity; and identify the relative merits and limitations of each of the five methods. All these goals are closely associated with the effects of thermomechanical noise and fluctuations on dynamic and thermodynamic properties. When a dissipative structure is coupled to its thermal reservoir, the structure will exhibit spontaneous random fluctuations (also known as thermomechanical noise) as specified by the FluctuationDissipation Theorem. Numerous theoretical and experimental studies have extensively studied the effects of thermomechanical noise on the dynamics, performance, and linearity of MEMS and NEMS (see, for instance, [18]-[23]), but this phenomenon has not yet been explored using atomistic simulations.

\section{Simulation Methodology}

Molecular dynamics simulations were performed using the large-scale atomic/molecular massively parallel simulator (LAMMPS) package [24]. Single-crystal materials were constructed by arranging atoms on a face-centered cubic (fcc) lattice with a lattice parameter of $3.53 \AA$ for nickel, and on a body-centered tetragonal (bct) lattice with a lattice parameter of $5.43 \AA$ for silicon. The interactions between atoms were modeled using the embedded-atom-method (EAM) potential for Ni [25], [26] and the Stillinger-Weber potential for Si [27]. A Cartesian coordinate system was attached to the structures with the $x, y$, and $z$ axes oriented along the [ $\left.\begin{array}{lll}1 & 0 & 0\end{array}\right],\left[\begin{array}{lll}0 & 1 & 0\end{array}\right]$, and $\left[\begin{array}{lll}0 & 0 & 1\end{array}\right]$ directions respectively. The dimensions of the $y-z$ cross-section were $4.2 \mathrm{~nm} \times 4.2 \mathrm{~nm}$ for $\mathrm{Ni}$ and $4.3 \mathrm{~nm} \times 4.3$ $\mathrm{nm}$ for Si, and the length $(L)$ along the [1 100$]$-direction ranged from $6.75 \mathrm{~nm}$ to $7.60 \mathrm{~nm}$ (Table I). In the axial direction, all structures were clamped at one end, and free at the other. In the lateral ( $y$ and $z$ ) directions, the boundaries were free for
TABLE I

GEOMETRIC AND MECHANICAL PROPERTIES OF THE NANORESONATORS

\begin{tabular}{lcccc}
\hline \hline Structure & $\begin{array}{c}\text { Length } \\
(L)\end{array}$ & Cross-Section & $\begin{array}{c}\text { Axial } \\
\text { Stiffness } \\
\left(k_{\mathrm{x}}\right)\end{array}$ & $\begin{array}{c}\text { Fundamental } \\
\text { Natural } \\
\text { Frequency } \\
\left(f_{0}\right)\end{array}$ \\
\hline Ni nanofilm & $6.75 \mathrm{~nm}$ & $4.2 \mathrm{~nm} \times 4.2 \mathrm{~nm}$ & $552 \mathrm{~N} / \mathrm{m}$ & $183 \mathrm{GHz}$ \\
Si nanofilm & $7.60 \mathrm{~nm}$ & $4.3 \mathrm{~nm} \times 4.3 \mathrm{~nm}$ & $352 \mathrm{~N} / \mathrm{m}$ & $255 \mathrm{GHz}$ \\
Ni nanowire & $6.75 \mathrm{~nm}$ & $4.2 \mathrm{~nm} \times 4.2 \mathrm{~nm}$ & $316 \mathrm{~N} / \mathrm{m}$ & $136 \mathrm{GHz}$ \\
Si nanowire & $7.60 \mathrm{~nm}$ & $4.3 \mathrm{~nm} \times 4.3 \mathrm{~nm}$ & $210 \mathrm{~N} / \mathrm{m}$ & $202 \mathrm{GHz}$ \\
\hline
\end{tabular}

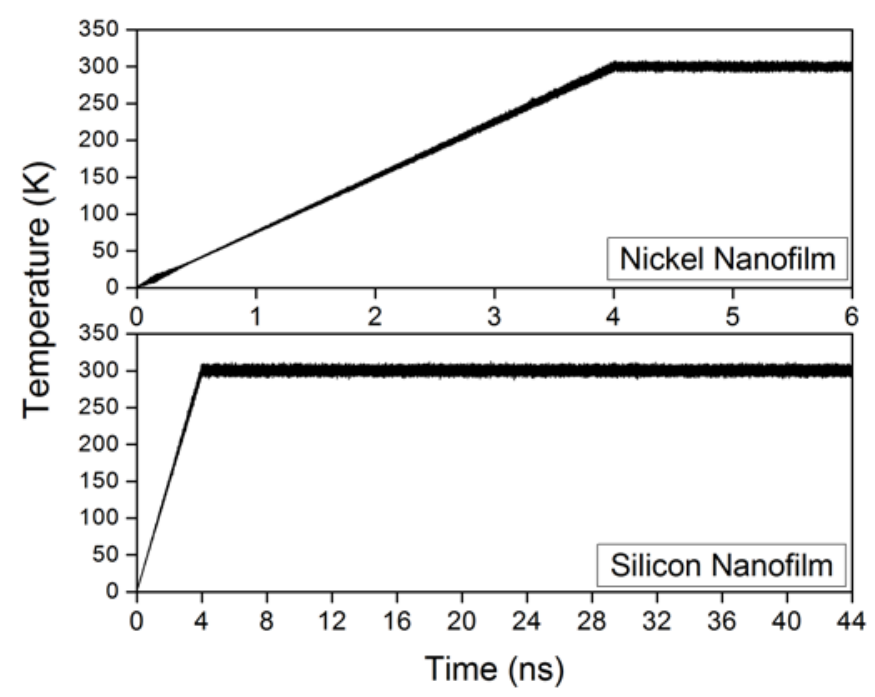

Fig. 1. Equilibration of the nanofilms at room temperature. The structures were heated from $1 \mathrm{~K}$ to $300 \mathrm{~K}$ over 3 to $5 \mathrm{~ns}$, and then further relaxed at $300 \mathrm{~K}$ for 2 ns in the case of nickel and for 40 ns in the case of silicon.

nanowires and periodic for nanofilms.

The equations of motion were integrated using the velocityVerlet algorithm [28] with a time step, $\tau_{\text {int }}=1 \mathrm{fs,} \mathrm{a} \mathrm{typical}$ value used in MD simulations of solids. The time step was selected as a trade-off between minimizing truncation errors during numerical integration and reducing computational cost. In addition, $\tau_{\text {int }}$ should be less than $10 \%$ of the shortest time period of atomic, molecular, or structural vibrations of the system [29]. All structures were evolved in time as an NVT ensemble using the Nosé-Hoover (NH) thermostat which is characterized by a time constant $\left(\tau_{N H}\right)$. In general, the time constant is chosen as a trade-off between minimizing fluctuations in temperature and the time required for attaining equilibrium. To identify a suitable value, parametric studies were performed by monitoring the potential energy, pressure, and temperature as functions of time for $\tau_{N H}$ ranging from 0.01 ps to 10 ps, and time constants of 0.1 ps and 0.01 ps were selected for $\mathrm{Ni}$ and $\mathrm{Si}$, respectively.

To attain thermodynamic equilibrium at room temperature, each structure was gradually heated from $1 \mathrm{~K}$ to $300 \mathrm{~K}$ over a duration ranging from 3 ns to $5 \mathrm{~ns}$, and then further relaxed at $300 \mathrm{~K}$ for $2 \mathrm{~ns}$ (for $\mathrm{Ni}$ ) and $40 \mathrm{~ns}$ (for $\mathrm{Si}$ ). Fig. 1 shows the temperature profile used for equilibrating the Ni nanofilm and Si nanofilm. After equilibration, the thermomechanical noise 


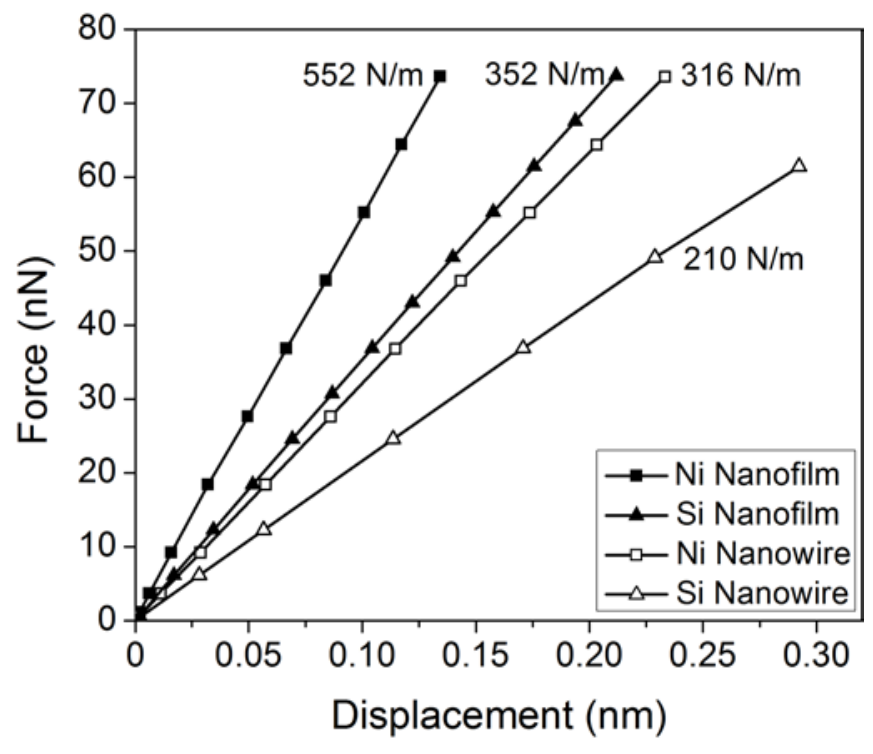

Fig. 2. Force-displacement curves obtained by simulating static tensile tests on nanowires and nanofilms of nickel and silicon. The axial stiffness was estimated by linear regression analysis.

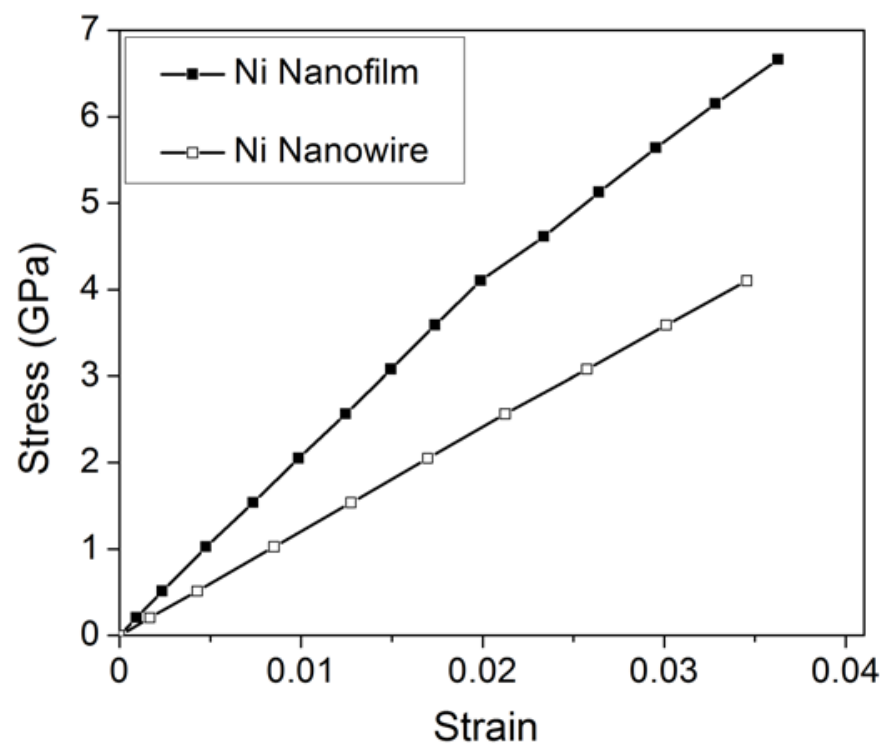

Fig. 3. Stress-strain curves obtained by simulating static tensile tests on the nickel nanostructures. The Young's modulus is $200 \mathrm{GPa}$ for the nanofilm and $119 \mathrm{GPa}$ for the nanowire.

spectrum was acquired by recording the spontaneous axial displacements of the atomic layer at the free end $(x=L)$ with respect to the mean center-of-mass coordinates. No external forces were applied during these simulations. The time-series was then analyzed in the frequency domain using the Fourier transform and the power spectral density (PSD). In both cases, the spectra exhibit characteristic peaks at the natural frequencies of longitudinal vibration. The first and most prominent peak was identified as the fundamental natural frequency.

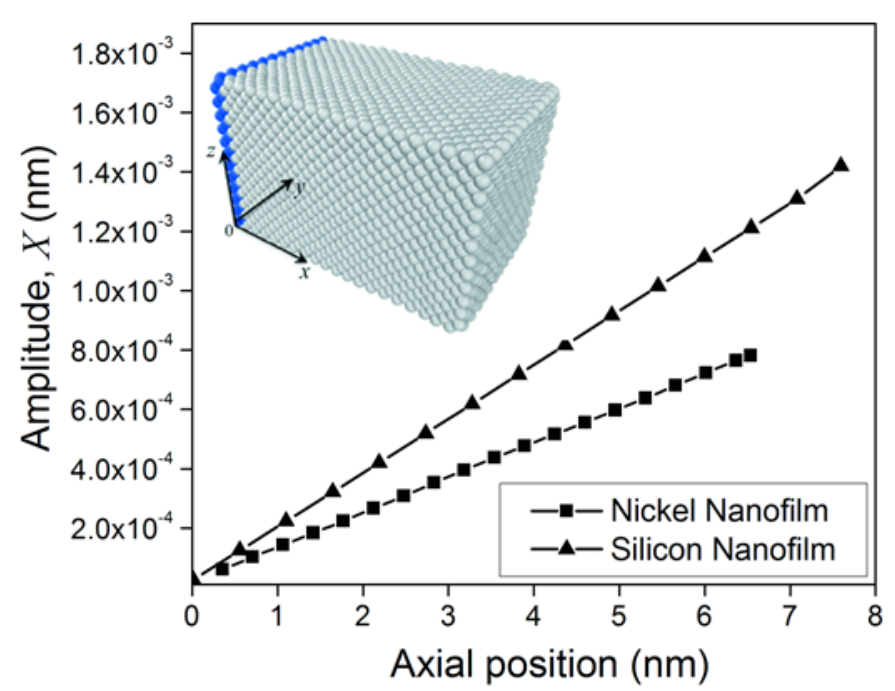

Fig. 4. Mode shape of the harmonic longitudinal oscillations of the nanofilms. The inset shows a schematic illustration of the face centered cubic (fcc) structure used to analyze damping in $\mathrm{Ni}$. The clamped end was simulated by fixing the displacements and velocities of two layers of atoms (shown in blue).

Subsequently, tensile tests were simulated by applying static forces at the free end of the structures and recording the corresponding displacements. The axial stiffness was obtained by a least-squares analysis of the elastic response; in all cases, the coefficient of regression exceeded 0.99. The Young's modulus and elastic limit were determined by transforming the data into axial stress and axial strain. Finally, the mode shape was determined by applying an axial harmonic force and recording the axial displacements at cross-sections along the length of the structures. The driving frequency was less than the fundamental natural frequency in these simulations.

Fig. 2 shows the force-displacement curves in the elastic regime for all four structures. The axial stiffness ranges from $210 \mathrm{~N} / \mathrm{m}$ to $552 \mathrm{~N} / \mathrm{m}$. The corresponding stress-strain curves are shown in Fig. 3 for the nickel nanofilm and nanowire. The former shows an elastic response followed by inelastic deformation, and the Young's modulus of $200 \mathrm{GPa}$ is consistent with bulk values and the properties of the EAM potential used for $\mathrm{Ni}$ [26]. The elastic limit (stress of $4 \mathrm{GPa}$ and strain of $2 \%$ ) is relatively large as a consequence of modeling the structures as ideal defect-free single crystals. In contrast, the nanowire deforms elastically before undergoing brittle fracture, and the Young's modulus drops to $119 \mathrm{GPa}$, which is in excellent agreement with previous MD simulations of the effects of free surfaces on the mechanical behavior of Ni nanowires [30]. Fig. 4 shows the mode shapes in the subresonant regime for the $\mathrm{Ni}$ nanofilm and $\mathrm{Si}$ nanofilm. To an excellent approximation, the displacement is a linear function of position and the axial strain is constant along the length of the structure, which is consistent with expectations based on an elementary model for the forced harmonic oscillations of a fixed-free elastic beam [15]. 


\section{DAmPING IN THE Sub-Resonant Regime}

In the sub-resonant regime, damping can be quantified in terms of the loss tangent and loss factor by applying an axial harmonic force, $F_{x}=F_{0} \sin \left(\omega_{d} t\right)$, at the free end of the structure and recording the velocity and displacement. Here, $F_{0}$ is the amplitude of the force, $\omega_{d}=2 \pi f_{d}$ is the angular driving frequency (with $f_{d}<f_{0}$ ), and $t$ is time. For all simulations, the steady-state axial response of the free end was well described by

$$
u(L, t)=\underbrace{X \sin \left(\omega_{d} t-\phi\right)}_{\text {Mechanical }}+\underbrace{\tilde{u}(L, t)}_{\text {Thermal }} .
$$

The first term is the harmonic mechanical response with amplitude $X$ and phase angle $\phi$, and the second term is the spontaneous random displacement due to thermomechanical noise. The phase angle was calculated in the frequencydomain using the Fourier transforms of the displacement and force. As a check, the results were verified by fitting the response in the time domain using linear regression analysis. The time-domain and frequency-domain estimates of $\tan \phi$ matched within $1 \%$. Using the same set of simulations, the loss factor was computed using the approach of Kunal and Aluru [15]. The work done per cycle of vibration is given by

$$
\Delta W=\left(\frac{2 \pi}{\omega_{d} \tau}\right) \Delta W_{\tau}=\left(\frac{2 \pi}{\omega_{d} \tau}\right) \int_{0}^{\tau} F_{0} \mathrm{v} \sin \left(\omega_{d} t\right) d t,
$$

where $\Delta W_{\tau}$ is the work done over time $\tau$, and $\mathrm{v}$ is the axial atomic velocity. The peak elastic energy is $W=0.5 k_{x} X^{2}$ and the amplitude $X$ was extracted from simulations using [15]

$$
X\left(\omega_{d}\right)=2 \times \frac{\operatorname{FFT}\langle u(L, t)\rangle}{n_{\text {data }}} .
$$

In this expression, $\langle u(L, t)\rangle$ is the average displacement of the atoms at the free end, and $n_{\text {data }}$ is the number of data points used in the Fast Fourier Transform (FFT).

\section{A. Response Regimes}

The displacement of the structure can be classified into three regimes: fluctuation-dominated response, linear mechanical response, and non-linear mechanical response. Fig. 5 illustrates the classification using the steady-state oscillations at $10 \mathrm{GHz}$ of the nickel nanofilm as a typical example. In the absence of the harmonic force $\left(F_{0}=0\right)$, the response is entirely due to thermomechanical noise with a root-mean-square (rms) value of $\sqrt{k_{B} T / k_{x}}=2.7 \times 10^{-3} \mathrm{~nm}$, where $k_{B}$ is Boltzmann's constant. For relatively small forces ( $F_{0}=0.2 \mathrm{nN}$ ), the harmonic response is of the same order as the thermomechanical noise $\left(\Delta W \sim k_{B} T\right)$. However, as $F_{0}$ increases further, the harmonic amplitude greatly exceeds the thermomechanical fluctuations and the displacement is dominated by the mechanical response. For $F_{0}=55 \mathrm{nN}$, the

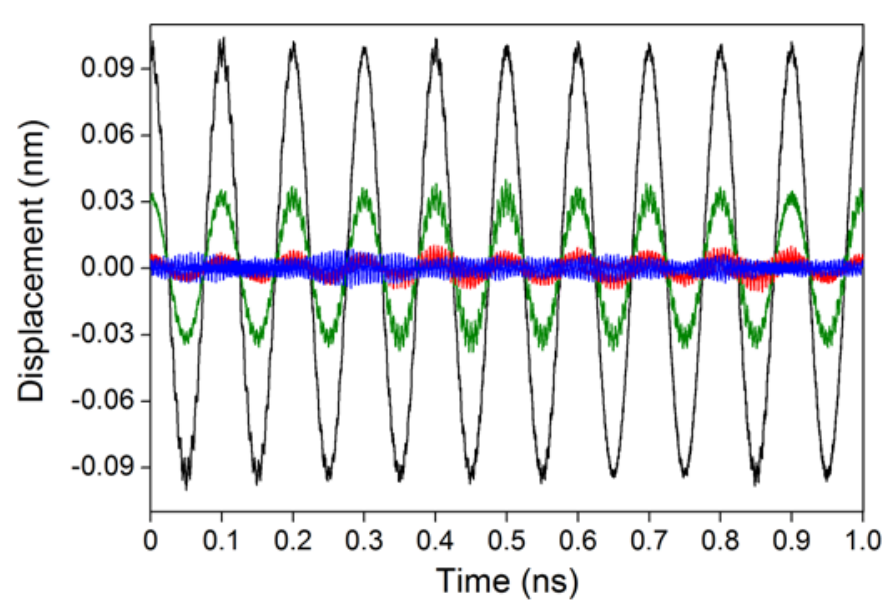

Fig. 5. Axial displacement as a function of time at the free end of the nickel nanofilm at $10 \mathrm{GHz}$. The blue curve shows the thermomechanical noise. The red, green, and black curves show the response when the structure is driven harmonically with force amplitudes of $2 \mathrm{nN}, 18 \mathrm{nN}$, and $55 \mathrm{nN}$, respectively.

harmonic amplitude is $\sim 0.1 \mathrm{~nm}$, which is more than an order of magnitude greater than the rms thermal noise.

In the fluctuation-dominated regime, the analysis of damping must account for fluctuations in thermodynamic quantities (entropy, work done, heat, internal energy, and dissipation). For a harmonic oscillator, the Steady-State Work Fluctuation Theorem can be expressed as [31]

$$
\frac{1}{\Sigma_{\tau}} \frac{p\left(\Delta W_{\tau}\right)}{p\left(-\Delta W_{\tau}\right)}=\exp \left(\frac{\Delta W_{\tau}}{k_{B} T}\right),
$$

where $p$ is the probability distribution function, $\tau$ is the time interval over which the work is calculated, and $\Sigma_{\tau}$ is a dimensionless factor that accounts for finite-time effects. Equation (4) has many intriguing consequences; for instance, depending on the statistical properties of the work distribution, there is a finite probability of observing trajectories for which the work done is negative (corresponding to entropy destruction) [31]. This topic is an active area of current research and only a few cases (including viscously-damped harmonic oscillators with a Gaussian distribution of $\Delta W_{\tau}$ ) have been studied thus far. Establishing finite-time corrections for systems dominated by anelastic or viscoelastic material damping, and for systems with non-Gaussian work distributions, are interesting open questions of fundamental scientific interest. However, from the viewpoint of estimating damping using classical MD, it is best to avoid the fluctuationdominated regime and focus instead on the mechanical regime.

Fig. 6 illustrates the difference between the fluctuationdominated regime and linear mechanical regime for the typical example of oscillations at $10 \mathrm{GHz}$ of the silicon nanofilm. Damping was calculated after every nanosecond: the symbols denote the loss factor and the lines indicate the loss tangent. In all cases, $\tan \phi$ differs from $\eta$ by less than $5 \%$. 
TABLE II

LINEAR DAMPING AT 300 K FOR NANOFILMS AND NANOWIRES OF NICKEL AND SILICON

\begin{tabular}{|c|c|c|c|c|c|c|}
\hline Structure & $\begin{array}{c}\text { Frequency, } \\
f(\mathrm{GHz})\end{array}$ & $\begin{array}{l}\text { Force, } F_{0} \\
\quad(\mathrm{nN})\end{array}$ & $\begin{array}{l}\text { Convergence } \\
\text { time, } \tau_{c} \text { (ns) }\end{array}$ & $\tan \phi$ & $\eta$ & $\frac{\tan \phi-\eta}{\tan \phi}$ \\
\hline \multirow{5}{*}{$\begin{array}{c}\text { Nickel } \\
\text { nanofilm }\end{array}$} & 10 & \multirow{5}{*}{55} & 80 & $8.67 \times 10^{-4}$ & $9.06 \times 10^{-4}$ & $4.5 \%$ \\
\hline & 15 & & 42 & $1.21 \times 10^{-3}$ & $1.27 \times 10^{-3}$ & $4.3 \%$ \\
\hline & 20 & & 30 & $1.50 \times 10^{-3}$ & $1.56 \times 10^{-3}$ & $4.0 \%$ \\
\hline & 25 & & 11 & $1.81 \times 10^{-3}$ & $1.87 \times 10^{-3}$ & $3.4 \%$ \\
\hline & 30 & & 14 & $2.05 \times 10^{-3}$ & $2.11 \times 10^{-3}$ & $2.6 \%$ \\
\hline \multirow{5}{*}{$\begin{array}{c}\text { Silicon } \\
\text { nanofilm }\end{array}$} & 10 & \multirow{5}{*}{49} & 122 & $2.44 \times 10^{-4}$ & $2.48 \times 10^{-4}$ & $1.7 \%$ \\
\hline & 15 & & 54 & $2.13 \times 10^{-4}$ & $2.16 \times 10^{-4}$ & $1.5 \%$ \\
\hline & 20 & & 76 & $2.00 \times 10^{-4}$ & $2.03 \times 10^{-4}$ & $1.4 \%$ \\
\hline & 25 & & 47 & $1.76 \times 10^{-4}$ & $1.77 \times 10^{-4}$ & $1.2 \%$ \\
\hline & 30 & & 51 & $1.62 \times 10^{-4}$ & $1.62 \times 10^{-4}$ & $0.0 \%$ \\
\hline \multirow{5}{*}{$\begin{array}{c}\text { Nickel } \\
\text { nanowire }\end{array}$} & 10 & \multirow{5}{*}{46} & 51 & $1.17 \times 10^{-3}$ & $1.15 \times 10^{-3}$ & $1.7 \%$ \\
\hline & 15 & & 27 & $1.64 \times 10^{-3}$ & $1.61 \times 10^{-3}$ & $1.8 \%$ \\
\hline & 20 & & 23 & $2.11 \times 10^{-3}$ & $2.06 \times 10^{-3}$ & $2.4 \%$ \\
\hline & 25 & & 19 & $2.57 \times 10^{-3}$ & $2.48 \times 10^{-3}$ & $3.5 \%$ \\
\hline & 30 & & 5 & $3.02 \times 10^{-3}$ & $2.87 \times 10^{-3}$ & $4.3 \%$ \\
\hline \multirow{4}{*}{$\begin{array}{c}\text { Silicon } \\
\text { nanowire }\end{array}$} & 10 & \multirow{4}{*}{37} & 38 & $4.24 \times 10^{-4}$ & $4.38 \times 10^{-4}$ & $3.2 \%$ \\
\hline & 15 & & 62 & $4.06 \times 10^{-4}$ & $4.18 \times 10^{-4}$ & $2.9 \%$ \\
\hline & 20 & & 56 & $3.82 \times 10^{-4}$ & $3.92 \times 10^{-4}$ & $2.6 \%$ \\
\hline & 25 & & 16 & $3.66 \times 10^{-4}$ & $3.74 \times 10^{-4}$ & $2.1 \%$ \\
\hline
\end{tabular}

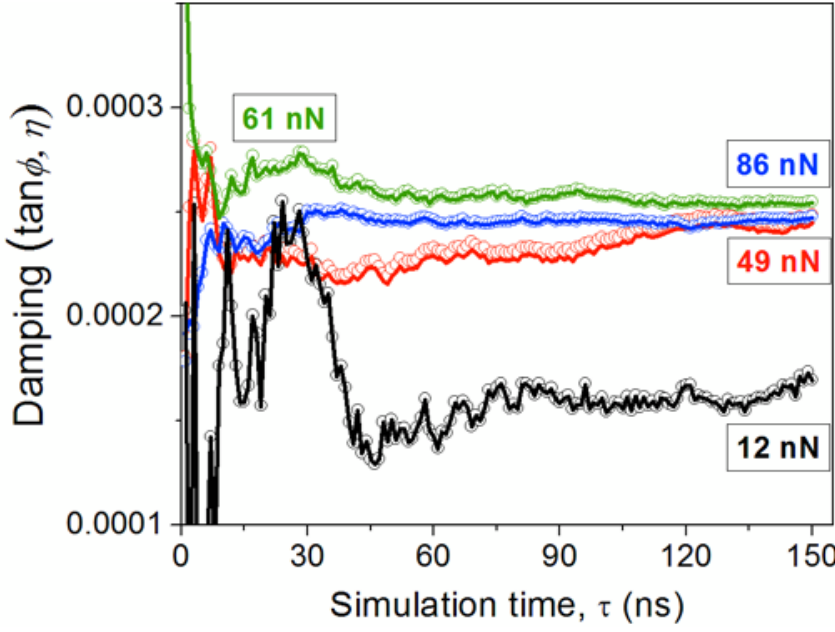

Fig. 6. Convergence behavior of damping for the longitudinal-mode oscillations at $10 \mathrm{GHz}$ of the silicon nanofilm. The open circles represent the loss factor $(\eta)$ and the lines represent the loss tangent $(\tan \phi$ ). Both measures were computed every nanosecond; in all cases, the difference between $\eta$ and $\tan \phi$ is less than $5 \%$.

In the fluctuation-dominated regime $\left(F_{0}=12 \mathrm{nN}\right)$, the damping fluctuates between $10^{-4}$ and $2.5 \times 10^{-4}$, and the simulations do not converge even after 150 ns of forced oscillation. In the mechanical regime $\left(F_{0}=49 \mathrm{nN}\right)$, the loss factor and loss tangent converge to $2.4 \times 10^{-4}$ after 122 ns. The convergence time, $\tau_{c}$, was defined as the simulation time beyond which the changes in $\tan \phi$ and $\eta$ were less than $3 \%$. Both measures remained unchanged when the force was increased further to $61 \mathrm{nN}$ and $86 \mathrm{nN}$, which satisfies the two criteria for linear damping: the loss factor and loss tangent are independent of the oscillation amplitude, and $\tan \phi=\eta[1]$, [32].

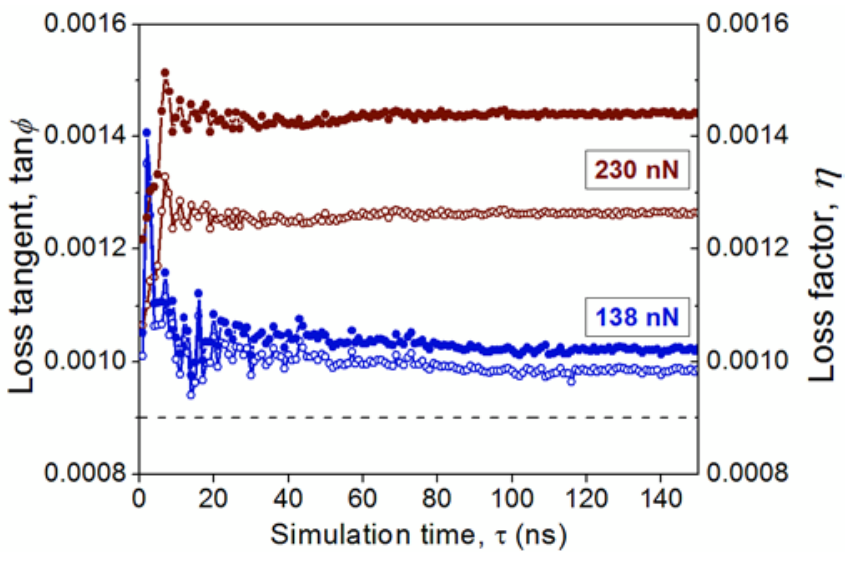

Fig. 7. Nonlinear damping in the longitudinal-mode oscillations at $10 \mathrm{GHz}$ of the nickel nanofilm. The loss factor and loss tangent are indicated by the open circles and closed circles, respectively.

The nanoresonator can be driven into the nonlinear mechanical regime by increasing the force beyond the linear limit. Fig. 7 shows a typical example of nonlinear damping in the longitudinal-mode oscillations at $10 \mathrm{GHz}$ of the nickel nanofilm. For this structure, the simulations converge in the linear regime with $\tan \phi=\eta=9 \times 10^{-4}$, as indicated by the dashed line in the graph. When the harmonic force exceeds the elastic limit of $92 \mathrm{nN}$, the damping exhibits both signatures of nonlinearity. Thus, for $F_{0}=230 \mathrm{nN}$, the loss tangent is $14 \%$ higher than the loss factor.

\section{B. Damping in the Linear Mechanical Regime}

Table II presents the full set of results for linear subresonant damping at room temperature for the longitudinal mode oscillations of nanowires and nanofilms of silicon and nickel. In all cases, damping was calculated in the linear Copyright (c) 2013 IEEE. Personal use is permitted. For any other purposes, permission must be obtained from the IEEE by emailing pubs-permissions@iee.org. 
mechanical regime. For each structure, damping was evaluated at several different frequencies ranging from $10 \mathrm{GHz}$ to 30 GHz. At each frequency, the convergence behavior was studied by computing the loss factor and loss tangent after each nanosecond for a total of $150 \mathrm{~ns}$ at different levels of harmonic excitation.

The convergence time is a function of several variables including the material, crystal structure, mode of oscillation, magnitude and mechanism of damping, frequency, force, stiffness, and the criterion used for convergence. All else being constant, the convergence time generally decreases as the harmonic force is increased because of a corresponding increase in the ratio of mechanical response to thermomechanical noise. Another general trend is a reduction of the convergence time at higher frequencies because the structure executes a greater number of oscillations per unit time. This trend is evident in Table II with the exception of the silicon nanowire at $10 \mathrm{GHz}$ and the silicon nanofilm at 20 $\mathrm{GHz}$. The reasons for these anomalies have yet to be identified. Finally, we note that the criterion of $3 \%$ is an arbitrary choice; $\tau_{c}$ will change significantly if other criteria are used for convergence.

\section{EstimAting $Q$ FROM THERMOMECHANICAL NOISE}

The power spectral density (PSD) of thermomechanical noise exhibits prominent peaks at the natural frequencies of oscillation [18]. For the three major classes of linear damping (viscous, viscoelastic, and anelastic), the first peak (corresponding to the fundamental natural frequency) is symmetric and well-approximated by a simple Lorentzian function given by [18], [33]

$$
S_{x}(f)=\frac{k_{B} T}{\pi k} \frac{\left(f_{0} / 2 Q\right)}{\left(f-f_{0}\right)^{2}+\left(f_{0} / 2 Q\right)^{2}},
$$

where $S_{x}$ denotes the PSD of axial displacement noise. Equation (5) can be used to estimate damping at the natural frequency by fitting the PSD to obtain the quality factor. Typically, the fit is performed by least-squares analysis of thermomechanical noise acquired over a finite time duration $\tau$ which will invariably introduce uncertainties in the estimate of the quality factor. To leading order, the standard deviation is given by [34]

$$
\sigma_{Q}=\sqrt{\frac{6 Q_{0}^{3}}{\pi \tau f_{0}}},
$$

where $Q_{0}$ is the mean value of the quality factor.

To implement this approach using MD simulations, the time-series of the spontaneous random fluctuations of the atomic layer at the free end $(x=L)$ were recorded at $300 \mathrm{~K}$ in the NVT ensemble without applying any external forces on the structure. The displacement was sampled at intervals of $1 \mathrm{fs}$ to ensure that the Nyquist frequency (that is, one-half of the sampling frequency) is significantly greater than the Copyright (c) 2013 IEEE. Personal use is permitted. For any other purposes, permission must be obtained from the IEEE by emailing pubs-permissions@iee.org.

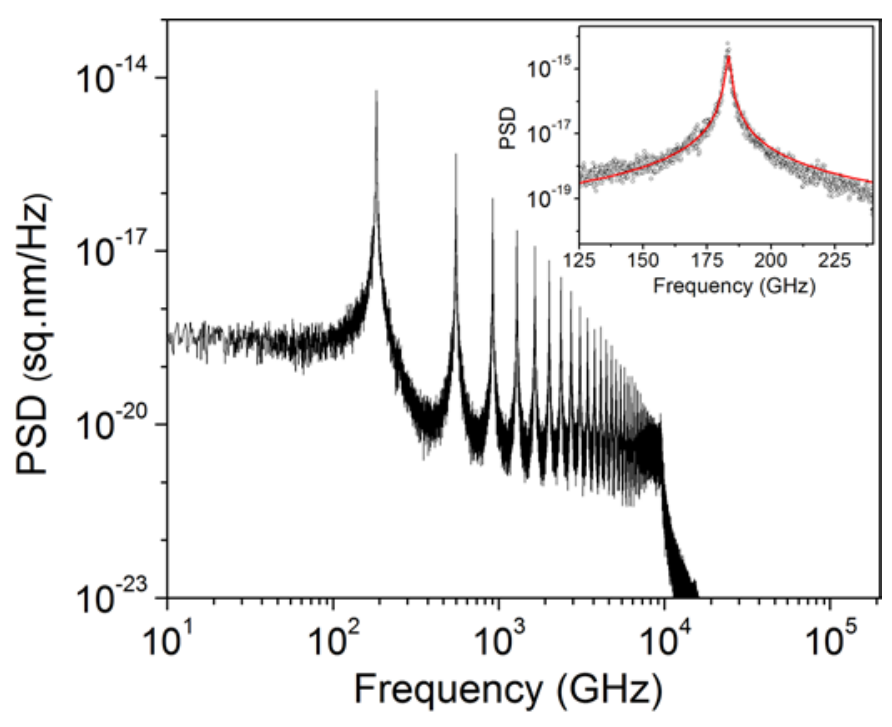

Fig. 8. Power spectral density (PSD) of the displacement noise for the nickel nanofilm. The inset shows the first peak corresponding to the fundamental longitudinal-mode at $183 \mathrm{GHz}$. The red line is the result of a least-squares fit to (5).

fundamental natural frequencies of the nanostructures. The thermomechanical noise was recorded for $\tau=154$ ns and then divided into five contiguous segments. For each segment, the PSD of the displacement noise was computed using the Welch periodogram technique implemented in MATLAB (The MathWorks, Inc., Natick, MA), and the first peak was fit to (5) by a weighted least-squares analysis using the LevenbergMarquardt algorithm to obtain the natural frequency and quality factor.

For the fundamental mode at $183 \mathrm{GHz}$ of the Ni nanofilm (Fig. 8), the analysis of the five noise segments resulted in estimates of 187, 174, 148, 219, and 186 for the quality factor. Hence, $Q_{0}=183$ and $\sigma_{Q}=25$. The latter is in good agreement with (6) which predicts a standard deviation of 20.3 (i.e., $11 \%$ of the mean).

\section{Estimating NONLINEAR DAMPING USING $Q$ AND $\delta$}

For linear systems, damping can be obtained by analyzing the steady-state harmonic oscillations in the frequency domain. As in the case of thermomechanical noise, the peak corresponding to the fundamental natural frequency can be fit to a Lorentzian function to obtain the quality factor. Alternately, the system can be subjected to an impulsive force and the freely decaying oscillations can be analyzed in the time domain to obtain the logarithmic decrement, $\delta$. For phenomenological models of viscous, viscoelastic, and anelastic damping, the two measures are related by $\delta=\pi Q^{-1}$ [1], and this relationship has been observed in MD simulations of linear damping in carbon nanotubes in the microcanonical NVE ensemble [10].

In stark contrast, our results indicate that the dynamics are strikingly different when nanomechanical structures are 
simulated in the canonical NVT ensemble. The use of a thermostat to couple the structure to its thermal bath, and the resultant thermomechanical noise, make it difficult to access the linear range. In other words, the structure can be driven into the nonlinear regime simply by requiring the harmonic response to be significantly greater than the thermal noise. Furthermore, the frequency response displayed the characteristic signatures of the Duffing-like nonlinearity, as also observed in experimental studies of nonlinear damping in micromechanical and nanomechanical resonators [20], [35][38].

For a Duffing oscillator, damping can be estimated in the frequency domain to obtain the quality factor [39]. Alternately, the free decay can be analyzed in the time domain to obtain the average log decrement as a function of oscillation amplitude [40]. Both methods are illustrated below for the fundamental mode oscillations at $183 \mathrm{GHz}$ of the nickel nanofilm.

\section{A. Duffing Nonlinearity at Resonance}

In the vicinity of the natural frequency, the nonlinear frequency response can be approximated by [20], [39]

$$
\frac{2 Q\left(f-f_{0}\right)}{f_{0}}=\frac{2}{\sqrt{3}} \frac{X^{2}}{X_{c}^{2}} \pm \sqrt{\frac{X_{p}^{2}}{X^{2}}-1},
$$

where $X_{p}$ is the peak amplitude and $X_{c}$ is the critical amplitude. The latter is the point of bifurcation (that is, the coordinate at which the slope of the amplitude-frequency curve is infinite) [20]. The backbone curve (that is, the graph of $X_{p}$ as a function of the peak frequency, $f_{p}$ ) is obtained by setting $X=X_{p}$. The term under the radical vanishes to give

$$
\frac{2 Q\left(f_{p}-f_{0}\right)}{f_{0}}=\frac{2}{\sqrt{3}} \frac{X_{p}^{2}}{X_{c}^{2}}
$$

The quality factor was estimated by applying harmonic axial forces (with amplitudes ranging from $0.09 \mathrm{nN}$ to $0.23 \mathrm{nN}$ ) and recording the steady-state mechanical response. The frequency response was examined to identify the critical amplitude (for this structure, $X_{c}=0.04 \mathrm{~nm}$ ) and the backbone curve was obtained from simulations and fit to (8) to obtain $f_{0}=183$ $\mathrm{GHz}$ and $Q=215$. Finally, the frequency response curves were computed using (7).

Fig. 9 shows the nonlinear frequency response for the nickel nanofilm. The spring softening nonlinearity was also observed in simulations of the silicon nanofilm. Material nonlinearities can be eliminated as the origin of this response because the oscillation amplitudes $(\leq 0.06 \mathrm{~nm})$ are an order of magnitude below the elastic limit. Similarly, dissipative nonlinearities can be neglected because the quality factor of 215 is in good agreement with the value $(183 \pm 25)$ obtained from the linear analysis of thermomechanical noise. By a process of elimination, and guided by an analysis of the mode shapes in the vicinity of resonance, we identify mode coupling

Copyright (c) 2013 IEEE. Personal use is permitted. For any other purposes, permission must be obtained from the IEEE by emailing pubs-permissions@iee.org.

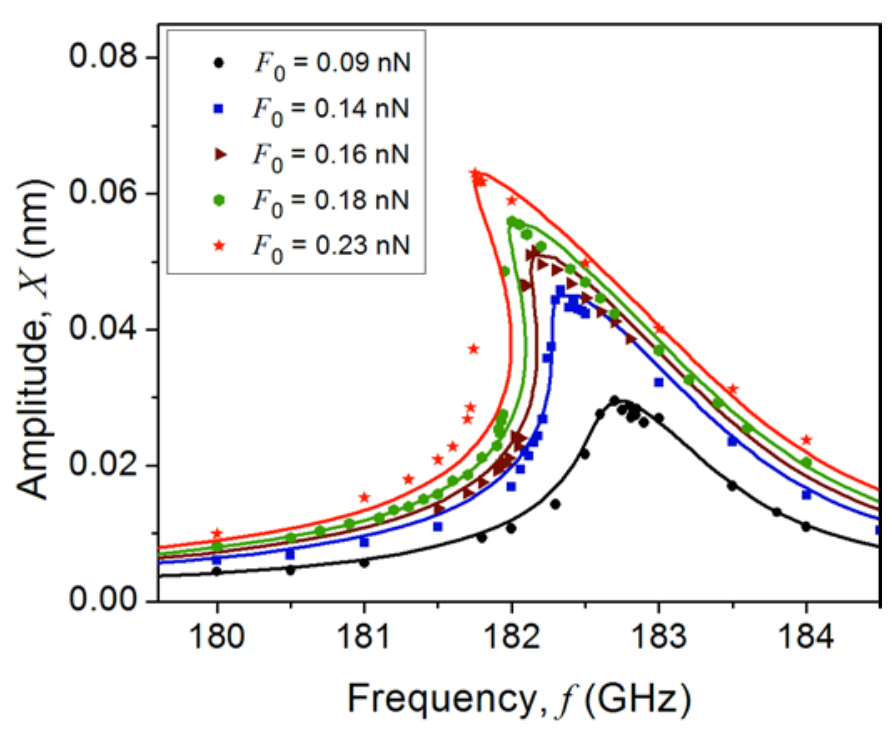

Fig. 9. Nonlinear frequency response for the nickel nanofilm. The symbols are the results of simulations and the curves were computed using (7) with $X_{c}=0.04 \mathrm{~nm}, f_{0}=183 \mathrm{GHz}$, and $Q=215$.

(specifically, a coupling of the longitudinal mode at $183 \mathrm{GHz}$ with torsional modes at $128 \mathrm{GHz}$ ) as the source of the Duffing nonlinearity. This conclusion is qualitatively supported by the literature on the nonlinear oscillations of fixed-free beams which suggests that spring softening can arise when new degrees-of-freedom are activated by mode coupling [41].

\section{B. Nonlinear Free Decay}

The free decay method was implemented by applying a harmonic axial force with amplitude of $0.09 \mathrm{nN}$ on the nickel nanofilm for 2 ns. The excitation frequency was close to, but less than, the fundamental natural frequency of $183 \mathrm{GHz}$. After the structure reached steady-state, the excitation was terminated and the free oscillations of the atomic layer at $x=L$ were recorded. In the first nanosecond of free oscillations, the amplitude reduced from $2.5 \times 10^{-2} \mathrm{~nm}$ to $5.2 \times 10^{-3} \mathrm{~nm}$. The free decay was segmented into timewindows, each 0.03 ns in duration, and then analyzed using FFT to determine the instantaneous decay frequency. This frequency varied between $180 \mathrm{GHz}$ and $185 \mathrm{GHz}$, which is a signature of the underlying nonlinear dynamics.

Finally, the damping was estimated by combining the decay signal $x(t)$ with its Hilbert transform $\mathrm{H}[x(t)]=\tilde{x}(t)$ to construct the analytic signal $z(t)=x(t)+i \tilde{x}(t)$ [40]. The log decrement was extracted by linear regression analysis of the slope of $\ln (A(t))$, where $A(t)=\sqrt{x^{2}(t)+\tilde{x}^{2}(t)}$ is the envelope signal [40]. The damping varied as a function of the oscillation amplitude (and hence as a function of the decay duration). For the full decay duration of $1 \mathrm{~ns}$, the log decrement $(\delta=0.015)$ is in good agreement with the frequency domain analysis $\left(\pi Q^{-1}=0.0146\right)$. 
TABLE III

COMPARISON OF THE LOSS FACTORS OF THE NI NANOFILM.

\begin{tabular}{lccc}
\hline \hline \multirow{2}{*}{ Frequency } & \multicolumn{3}{c}{ Loss factor } \\
\cline { 2 - 4 } & $\begin{array}{c}\text { Present } \\
\text { study }\end{array}$ & Ref.[15] & Difference \\
\hline $10 \mathrm{GHz}$ & $\begin{array}{c}9.06 \times 10^{-4} \\
(80 \mathrm{~ns})\end{array}$ & $\begin{array}{c}6.90 \times 10^{-4} \\
(30 \mathrm{~ns})\end{array}$ & $23.8 \%$ \\
$15 \mathrm{GHz}$ & $\begin{array}{c}1.27 \times 10^{-3} \\
(42 \mathrm{~ns})\end{array}$ & $\begin{array}{c}1.08 \times 10^{-3} \\
(30 \mathrm{~ns})\end{array}$ & $14.9 \%$ \\
$20 \mathrm{GHz}$ & $\begin{array}{c}1.56 \times 10^{-3} \\
(30 \mathrm{~ns})\end{array}$ & $\begin{array}{c}1.37 \times 10^{-3} \\
(30 \mathrm{~ns})\end{array}$ & $12.1 \%$ \\
$25 \mathrm{GHz}$ & $\begin{array}{c}1.87 \times 10^{-3} \\
(11 \mathrm{~ns})\end{array}$ & $\begin{array}{c}1.67 \times 10^{-3} \\
(30 \mathrm{~ns})\end{array}$ & $10.7 \%$ \\
$30 \mathrm{GHz}$ & $2.11 \times 10^{-3}$ & $1.92 \times 10^{-3}$ & $9.0 \%$ \\
& $(14 \mathrm{~ns})$ & $(30 \mathrm{~ns})$ & \\
\hline
\end{tabular}

The numbers in parentheses denote the simulation time.

\section{DISCUSSION}

In this paper, we explored five different methods for computing damping in a single mode of vibration under isothermal conditions using atomistic simulations. The methods were demonstrated by using classical molecular dynamics to simulate the fundamental mode of longitudinal oscillations of nanowires and nanofilms of two materials (silicon and nickel) in the canonical NVT ensemble, and damping was calculated at $300 \mathrm{~K}$ by controlling the temperature with the Nosé-Hoover thermostat.

\section{A. Comparison of Methods}

For all methods, damping was estimated without recourse to macroscale (or continuum-based) concepts. Instead, the analysis is based on simple dynamic models for the linear and nonlinear oscillations of a system with a single degree-offreedom. The choice of the dynamic model was guided by the response observed in the simulations, and by following the dictum of selecting the simplest model that can provide the required level of detail and insight. For instance, the equations used for estimating the loss tangent and loss factor were selected after observing the linear force-displacement curve (Fig. 2) and steady-state time-harmonic oscillations (Fig. 5) in the sub-resonant regime. Similarly, the use of the Duffing model was guided by the spring-softening nonlinearity observed at resonance.

In the sub-resonant regime, damping can be estimated by using the loss tangent and loss factor. No assumptions are made about the nature, magnitude, or mechanisms of dissipation. The same structure can be used to simulate damping at several different frequencies below the fundamental natural frequency. Further, both measures can be computed using the same set of simulations with little incremental cost. It is useful to do so because the relative values of $\tan \phi$ and $\eta$ can be used to assess linearity. In general, however, simulating damping in the sub-resonant regime is expensive because of the cost associated with assessing convergence and linearity.

Analysis of thermomechanical noise is the method of choice for obtaining the quality factor at resonance. Simple formulas are available for estimating the mean and standard deviation of the quality factor. Equation (5) can be applied to all major Copyright (c) 2013 IEEE. Personal use is permitted. For any other purposes, permission must be obtained from the IEEE by emailing pubs-permissions@ieee.org. classes of linear dissipation (anelastic, viscoelastic, and viscous) without any assumptions about the underlying mechanisms. In principle, the technique can be extended to extract the quality factors of the higher modes of oscillations by using modal coordinates to analyze the PSD [42]. However, the modal approach requires assumptions about the mode shapes and spatial distribution of damping [42, 43].

For nanomechanical resonators, it is usually necessary to account for nonlinearities while analyzing the frequency response at resonance. In our simulations, the response was accurately captured by the Duffing equation which assumes linear dissipation with a damping force that is proportional to velocity [39]. For structures with significant nonlinear dissipation, it is necessary to use higher-order models to analyze the response. In recent studies of MEMS and NEMS, a nonlinear damping coefficient has been introduced into the equation of motion in a term that contains the product of the velocity and the square of the displacement [37, 38]. For this model, simple closed-form expressions (analogous to (7) and (8)) are available for extracting the nonlinear damping coefficient by analyzing the frequency response in the vicinity of resonance [37, 44].

\section{B. Validation of Results}

As a check on our simulations, the loss factors for the $\mathrm{Ni}$ nanofilm (Table II) were compared with previous results presented in Ref. [15]. The structural dimensions and several simulation parameters (including the EAM potential, integration time step, and the thermostat time constant) are the same in both studies, but the simulation times are significantly different at certain frequencies, as noted parenthetically in Table III. The two studies are in good agreement with differences ranging from $9 \%$ to $24 \%$.

A direct comparison with experiments is not currently possible because of a lack of measurements of damping at the ultrahigh frequencies under consideration. However, an indirect comparison can be made by using a simple model for Akhiezer damping to link simulations with experiments. Kunal and Aluru [15] have shown that the frequency dependence of damping in Table III is consistent with expectations based on Akhiezer damping with a single relaxation time. Furthermore, the relaxation time obtained from simulations matches experimental data. Our preliminary simulations suggest that the same is true for single-crystal silicon. We are currently refining this analysis with extensive MD simulations of damping in the longitudinal-mode oscillations of nanoresonators constructed using single-crystal and amorphous silicon.

\section{REFERENCES}

[1] A. S. Nowick and B. S. Berry, Anelastic Relaxation in Crystalline Solids. New York: Academic, 1972.

[2] R. Lakes, Viscoelastic Materials. UK: Cambridge University Press, 2009.

[3] J. Woodhouse, "Linear damping models for structural vibration," $J$. Sound Vib., vol. 215, pp. 547-569, Aug. 1998.

[4] S. Joshi, S. Hung, and S. Vengallatore, "Design strategies for controlling damping in micromechanical and nanomechanical resonators," EPJ Techniques and Instrumentation, vol. 1, article no. 5, May 2014. 
[5] D. Frenkel and B. Smit, Understanding Molecular Simulations: From Algorithms to Applications, NY: Academic Press, 2001.

[6] R. Rudd and J. Broughton, "Atomistic simulation of MEMS resonators through the coupling of length scales," J. Model. Simul. Microsyst., vol. 1, no. 1, pp. 29-38, Feb. 1999.

[7] W. Guo, Y. Guo, H. Gao, Q. Zheng, and W. Zhong, "Energy dissipation in gigahertz oscillators from multiwalled carbon nanotubes," Phys. Rev. Lett., vol. 91, no. 12, 125501-1-125501-4, Sep. 2003.

[8] H. Jiang, M. -F. Yu, B. Liu, and Y. Huang, "Intrinsic energy loss mechanisms in a cantilevered carbon nanotube beam oscillator," Phys. Rev. Lett., vol. 93, no. 18, 185501-1-185501-4, Oct. 2004.

[9] S. Akita, S. Sawaya, and Y. Nakayama, "Energy loss of carbon nanotube cantilevers for mechanical vibration," Jap. J. Appl. Phys., vol. 46, pp. 6295-6298, Sep. 2007.

[10] A. K. Vallabhaneni, X. Ruan, J. F. Rhoads, and J. Murthy, “A band-pass filter approach within molecular dynamics for the prediction of intrinsic quality factors of nanoresonators,” J. Appl. Phys., vol. 112, 074301, Oct. 2012.

[11] Z. Zhou, V. K. Vasudevan, and D. Qian, "Energy loss in carbon nanotube beam oscillators due to anelastic relaxation,” J. Eng. Mat. Tech., vol. 134, no. 3, pp. 031005-1-031005-8, May 2012.

[12] K. Kunal and N. R. Aluru, "Intrinsic loss due to unstable modes in graphene," Nanotechnology, vol. 24, 275701, Apr. 2013.

[13] D. Midtvedt, A. Croy, A. Isacsson, Z. Qi, and H. S. Park, "Fermi-PastaUlam physics with nanomechanical graphene resonators: intrinsic relaxation and thermalization from flexural mode coupling," Phys. Rev. Lett., vol. 112, 145503, Apr. 2014.

[14] H. F. Zhan and Y. T. Gu, "A fundamental numerical and theoretical study for the vibrational properties of nanowires," J. Appl. Phys., vol. 111, pp. 124303-1-124303-8, Jun. 2012.

[15] K. Kunal and N. R. Aluru, "Akhiezer damping in nanostructures," Phys. Rev. B., vol. 84, pp. 245450-1-245450-8, Dec. 2011.

[16] W. G. Hoover, "Canonical dynamics: equilibrium phase-space distributions,” Phys. Rev. A, vol. 31, no. 3, pp. 1695-1697, Mar. 1985.

[17] S. Nosé, "A unified formulation of the constant temperature moleculardynamics methods," J. Chem. Phys., vol. 81, no. 1, pp. 511-519, Jul. 1984

[18] P. R. Saulson, "Thermal noise in mechanical experiments", Phys. Rev. D, vol. 42, pp. 2437-2445, Oct. 1990.

[19] T. B. Gabrielson, "Mechanical-thermal noise in micromachined acoustic and vibration sensors," IEEE Trans. Elec. Dev., vol. 40, pp. 903-909, May 1993.

[20] H. W. Ch. Postma, I. Kozinsky, A. Husain, and M. L. Roukes, "Dynamic range of nanotube- and nanowire-based electromechanical systems,” Appl. Phys. Lett., vol. 86, pp. 223105-1-223105-3, May 2005.

[21] J. Gieseler, L. Novotny, and R. Quidant, "Thermal nonlinearities in a nanomechanical oscillator,” Nature Phys., vol. 9, pp. 806-810, Nov. 2013.

[22] A. W. Barnard, V. Sazonova, A. M. van der Zande, and P. L. McEuen, "Fluctuation broadening in carbon nanotube resonators," Proc. Nat. Acad. Sci., vol. 109, pp. 19093-19096, Oct. 2012.

[23] S. D. Senturia, Microsystem Design. MA: Kluwer, 2001.

[24] S. Plimpton, "Fast parallel algorithms for short-range molecular dynamics," J. Comput. Phys., vol. 117, pp. 1-19, Mar. 1995.

[25] M. S. Daw and M. I. Baskes, "Embedded-atom method: derivation and application to impurities, surfaces, and other defects in metals," Phys. Rev. B, vol. 29, pp. 6443-6453, Jun. 1984.

[26] S. M. Foiles, M. I. Baskes, and M. S. Daw, "Embedded-atom-method functions for the fcc metals $\mathrm{Cu}, \mathrm{Ag}, \mathrm{Au}, \mathrm{Ni}, \mathrm{Pd}, \mathrm{Pt}$, and their alloys," Phys. Rev. B, vol. 33, pp. 7983-7991, Jun. 1986.

[27] F. H. Stillinger and T. A. Weber, "Computer simulation of local order in condensed phases of silicon,” Phys. Rev. B., vol. 31, pp. 5262-5271, Apr. 1985.

[28] W. C. Swope, H. C. Andersen, P. H. Berens, K. R. Wilson, “A computer simulation method for the calculation of equilibrium constants for the formation of physical clusters of molecules: application to small water clusters,” J. Chem. Phys., vol. 76, pp. 637-649, Sep. 1982.

[29] A. Gro $\beta$, Theoretical Surface Science: A Microscopic Perspective, NY: Springer-Verlag, 2007.

[30] P. S. Branicio and J.-P. Rino, "Large deformation and amorphization of Ni nanowires under uniaxial strain: A molecular dynamics study,” Phys. Rev. B., vol. 62, pp.16950-16955, Dec. 2000.
[31] F. Douarche, S. Joubaud, N. B. Garnier, A. Petrosyan, and S. Ciliberto, "Work fluctuation theorem for harmonic oscillators," Phys. Rev. Lett., vol. 97, 140603, Oct. 2006.

[32] Z. Nourmohammadi, Thermoelastic Damping in Micromechanical and Nanomechanical Resonators, Ph.D. dissertation, Dept. Mech. Eng., McGill University, Montreal, QC, Canada, Nov. 2014.

[33] O. Kuter-Arnebeck, A. Labuda, S. Joshi, K. Das, and S. Vengallatore, "Estimating damping in microresonators by measuring thermomechanical noise using laser Doppler vibrometry," J. Micromech. Syst., vol. 23, pp. 592-599, Jun. 2014.

[34] J. E. Sader, B. D. Hughes, J. A. Sanelli, and E. J. Bieske, "Effect of multiplicative noise on least-squares parameter estimation with applications to the atomic force microscope,” Rev. Sci. Instrum., vol. 83, 055106, 2012.

[35] V. Kaajakari, T. Mattila, A. Oja, and H. Seppa, "Nonlinear limits for single-crystal silicon microresonators," J. Micromech. Syst., vol 13, pp. 715-724, Oct. 2004.

[36] J. F. Rhoads, S. W. Shaw and K. L. Turner, "Nonlinear dynamics and its applications in micro- and nanoresonators," J. Dynamic Systems, Measurement and Control, vol. 132, pp. 034001-1-034001-14, Apr. 2010.

[37] A. Eichler, J. Moser, J. Chaste, M. Zdrojek, I. Wilson-Rae and A. Bachtold, "Nonlinear damping in mechanical resonators made from carbon nanotubes and graphene," Nature Nanotechnology, vol. 6, pp.339-342, May 2011.

[38] S. Zaitsev, O. Shtempluck, E. Buks, and O. Gottlieb, "Nonlinear damping in a micromechanical oscillator," Nonlinear Dyn. Vol. 67, pp. 859-883, Apr. 2012.

[39] A. H. Nayfeh and D. T. Mook, Nonlinear Oscillations. New York: Wiley, 2004.

[40] M. Feldman, Hilbert Transform Applications in Mechanical Vibration. New York: Wiley, 2011.

[41] A. H. Nayfeh and P. F. Pai, Linear and Nonlinear Structural Mechanics, New York: Wiley, 2004.

[42] M. V. Salapaka, H. S. Bergh, J. Lai, A. Majumdar, and E. McFarland, "Multi-mode noise analysis of cantilevers for scanning probe microscopy,” J. Appl. Phys., vol. 81, pp. 2480-2487, Mar. 1997.

[43] Y. Levin, "Internal thermal noise in the LIGO test masses: a direct approach,” Phys. Rev. D, vol. 57, pp. 659-663, Jan. 1998.

[44] M. Imboden, O. Williams, and P. Mohanty, "Nonlinear dissipation in diamond nanomechanical resonators," Appl. Phys. Lett., vol. 102, 103502, 2013.

Zahra Nourmohammadi received the B.Eng. degree in mechanical engineering from the Isfahan University of Technology, Isfahan, Iran, in 2005, the M.Eng. degree in mechanical engineering from Sharif University of Technology, Tehran, Iran, in 2008, and the Ph.D. degree in mechanical engineering from McGill University, Montreal, QC, Canada, in 2015.

Her research focuses on exploring damping in micro- and nanomechanical structures using continuum analysis and atomistic simulations.

Sankha Mukherjee received the B.Eng. degree in metallurgy and materials engineering from the Bengal Engineering and Science University, Shibpur, India, in 2007, and the M.Tech. degree in materials science from the Indian Institute of Technology-Bombay, Mumbai, India, in 2010. He is currently working towards the Ph.D. degree in the Department of Mechanical Engineering, McGill University, Montreal, QC, Canada. His research focuses on the analysis of damping in micro- and nanomechanical structures using measurements and atomistic simulations.

Mr. Mukherjee is a recipient of the McGill Engineering Doctoral Award (MEDA) from McGill University. 
Surabhi Joshi received the B.Eng. and M.Eng. degrees in mechanical engineering from McGill University, Montreal, QC, Canada, in 2011 and 2013, respectively. She is currently working as a Research Associate at the same institution. Her research focuses on damping and dissipation in micro- and nanomechanical systems.

Ms. Joshi is a recipient of the Provost's Graduate Fellowship from McGill University.

Jun Song received the B.Sc. degree in mechanical engineering from the University of Science and Technology of China, Hefei, China, in 2003, and the Ph.D. degree in mechanical and aerospace engineering from Princeton University, Princeton, USA, in 2008.

He is currently an Assistant Professor with the Department of Mining and Materials Engineering, McGill University. His current research interests include multiscale modeling of materials, hydrogen embrittlement, and defects-induced physics and mechanics in low-dimensional nanomaterials.
Srikar Vengallatore received the B.Tech. degree (with honours) in metallurgical engineering from the Indian Institute of Technology, Banaras Hindu University, Varanasi, India, in 1994, and the Ph.D. degree in materials science from the Massachusetts Institute of Technology (MIT), Cambridge, MA, in 1999.

After postdoctoral research at MIT from 1999 to 2003, he joined McGill University, Montreal, QC, Canada, where he is currently an Associate Professor in the Department of Mechanical Engineering. His current research interests include the nanomechanical behavior of materials and structures, and the design of microsystems for sensing, energy harvesting, and portable power generation.

Dr. Vengallatore held a Canada Research Chair from 2004 to 2014. He received the Class of '44 Award for Outstanding Teaching in 2007 and the Early Career Research Excellence Award in 2009. 